\title{
Development of a Corrosion Potential Measuring System Based on the Generalization of DACS Physical Scale Modeling
}

\author{
Dalei Song ${ }^{1}$, Xinjian $\mathrm{Fan}^{1}$, Xueyan $\mathrm{Ma}^{1}$, Weiguo $\mathrm{Shi}^{2, \mathrm{a}}$ and Xiangdong Wang ${ }^{1}$ \\ 1 Ocean University of China, Qingdao,266100, China \\ ${ }^{2}$ CNOOC Changzhou Paint \& Coating Industry Research Institute Co., LTD, Tanggu, 300452, China
}

\begin{abstract}
A feasible method in evaluating the protection effect and corrosion state of marine cathodic protection (CP) systems is collecting sufficient electric potential data around a submarine pipeline and then establishing the mapping relations between these data and corrosion states of pipelines. However, it is difficult for scientists and researchers to obtain those data accurately due to the harsh marine environments and absence of dedicated potential measurement device. In this paper, to alleviate these two problems, firstly, the theory of dimension and conductivity scaling (DACS) physical scale modeling of marine impressed current cathodic protection (ICCP) systems is generalized to marine CP systems, secondly, a potential measurement device is developed specially and analogue experiment is designed according to DACS physical scale modeling to verify the feasibility of the measuring system. The experimental results show that 92 percent of the measurement errors are less than $0.25 \mathrm{mv}$, thereby providing an economical and feasible measuring system to get electric potential data around an actual submarine pipeline under CP.
\end{abstract}

\section{Introduction}

Maintaining the integrity of the many thousands of miles of subsea pipeline of oil and gas is of the utmost importance, which is not only from an investment standpoint, but also from a public safety and environmental protection point of view. Currently, the most widely used anti-corrosion method is adding anticorrosion layer together with ICCP or CP system [1]. CP system has always played a prominent role in controlling external corrosion on many of these pipelines. For the subsea pipeline under $\mathrm{CP}$, the potential distribution data around a pipeline are valuable information to predict some crucial issues associated with seawater corrosion, such as coating defect problem, optimization design and evaluation of a CP system and so on. Therefore, how to get the potential data of a long subsea pipeline in harsh marine environment has been an important and challenging task for scientists and researchers.

Currently there are three methods to get potential data to establish the corrosion electric field modelling for scientific research. The first one is field measurement by diver or using remotely operated vehicle (ROV) [2, 3], which is hard to implement and very time consuming, thus being not a wise choice for potential data collection. The second one is numerical simulation based on finite element method (FEM) or boundary element method (BEM). Marcassoli and Bonetti employed FEM to simulate the potential distribution around pipeline under different degrees of coating damage [4]. However, these methods rely heavily on the experimental data and the polarization characteristic of the materials we used, which are generally difficult to obtain in practice. The third one is using laboratory simulation platform. It has been proved that the DACS physical scale modelling is a feasible approach for the design and optimization of ICCP system of a ship or submarine pipelines [5] and is also being used as a practical and flexible tool for study of corrosion [6-8]. However before we can acquire believable data of corrosion potential around a subsea pipeline under CP system by using DACS as the theoretical basis, we should solve two key issues: the first is that the method of designing a DACS physical scale modeling under ICCP cannot be used to design a DACS physical scale model under CP directly, the other is that we need a dedicated potential measuring system which can adapt to the complex physical and chemical environment of electrolyte.

To overcome the problems mentioned above, the theory of DACS physical scale modeling of marine ICCP systems is generalized to marine $\mathrm{CP}$ systems in this paper and a dedicated potential measuring system is developed according to the electrochemical properties of $\mathrm{Ag} / \mathrm{AgX}$ solid solution reference electrode in marine environment. Finally, analogue experiment based on DACS and simulations by FEM are performed to verify the effectiveness of the developed measuring system.

\section{Generalization of DACS physical scale model}

a Corresponding author: fxjouc@sina.com 
Physical scale model provides a method that can narrow down a prototype with big size in practical environment to an experimental model with smaller size based on correlation principle while keeping their characteristic parameters matched with each other. In the late twentieth century Ditchfield and Mcgrath strictly derived a theoretical transform for DACS physical scale model of ICCP systems [9], and they presented the corresponding relationships of characteristic parameters of electric field between model and prototype as showed in Table 1 ..

Table 1. The electromagnetic relation and dimensions relation between prototype and model. Noted that $p$ is the linear scaling factor, $\alpha$ and $\beta$ are arbitrary constants..

\begin{tabular}{|l|c|c|}
\hline Quantity & Prototype & Model \\
\hline Length & $L$ & $L^{\prime}=L / p$ \\
\hline Electric field & $E$ & $E^{\prime}=\alpha E$ \\
\hline Conductivity & $\sigma$ & $\sigma^{\prime}=\sigma / p$ \\
\hline Voltage & $V$ & $V^{\prime}=V / \alpha p$ \\
\hline Current density & $J$ & $J^{\prime}=p J / \beta$ \\
\hline
\end{tabular}

Since both of ICCP system and CP system prevent their cathodes from being corroded by providing current, the relationships between model and prototype showed in Table 1 is also valid for CP system. So for a CP system, according to those known relationships, we can also derive necessary conditions which guaranteeing the distributions of current density and potential of the model is proportional to those of the prototype.

As is shown in Table 1 we can know that if the following conditions are satisfied simultaneously:

$$
L^{\prime}=L / p, \sigma^{\prime}=\sigma / p, \alpha=1 / p, \beta=p
$$

Then conclusion that the potentials and the electric current densities of corresponding position in model and prototype are equal can be given as:

$$
V=V^{\prime}, \quad J=J^{\prime}
$$

Supposing that $U$ denotes potential drop, $d$ denotes distance, $A$ denotes cross-sectional area of current in prototype, similarly, $U^{\prime}, d^{\prime}, A^{\prime}$ in model, and $d / d^{\prime}=p$ is satisfied, then according to Ohm law, the potential drop in a certain distance ( $d$ in prototype and $d^{\prime}$ in model) can be represented as:

$$
\begin{gathered}
U=I d / \sigma A=J(d / \sigma)=(J d) / \sigma \\
U^{\prime}=I^{\prime} d^{\prime} / \sigma^{\prime} A^{\prime}=J^{\prime}\left(d^{\prime} / \sigma^{\prime}\right)=\left(J^{\prime} d^{\prime}\right) / \sigma^{\prime}
\end{gathered}
$$

Considering the relationship in Table 1 and equation (1), (3), .4) we can get:

$$
U=U^{\prime}, I=\left(p^{2} I^{\prime}\right)
$$

Hence, if we want to design a model system that is proportional to the prototype system, the provided current by the designed anodes of the model system should satisfy with equation (5).

Through searching on design criterion of anode, we can obtain the formula for calculating contact resistance $R$ of plate-shaped anode and formula for calculating electric current generated by anode as follows[10]:

$$
R=0.315 /(\sigma \times \sqrt{\mathrm{S}}), I=\Delta E / R
$$

where $S$ denotes the exposed area of anode, $I$ denotes the current produced by anode, and $\nabla E$ denotes the driving voltage of anode and is a constant decided by material of anode. Finally, combining equations (1), (5), (6) we can arrive at the conclusion that exposed area of anodes in model (named $S^{\prime}$ ) and prototype (named $S$ ) should satisfy with:

$$
\frac{S}{S^{\prime}}=\frac{I^{2} \times \sigma^{\prime 2}}{I^{\prime 2} \times \sigma^{2}}=p^{2}
$$

In a word, to ensure the effectiveness of generalization of DACS physical scale model from ICCP to $\mathrm{CP}$, we should narrow down the exposed area of anode according to the linear scaling factor $p$. For plate-shaped anodes we should guarantee equation (7) be tenable. For anodes having other shapes we can get their corresponding expression of contact resistance and deduce the corresponding equations of $S$ and $S$.

\section{Development of the measuring system}

\subsection{Selection and pre-treatment of the potentiometric sensor}

Because reference electrode is the key component for measuring or detecting the potential of seawater media of a CP system, its performance directly effect on the accuracy of measuring system. Hence, high precision and stability reference electrode should be used to measure protection potential or some other electrochemical parameters of a CP system of subsea pipelines in seawater. $\mathrm{Ag} / \mathrm{AgX}$ solid solution reference electrode is regarded as the most ideal reference electrode in seawater for its high accuracy and good reproducibility especially $[11,12]$.

It is noted that before measurement, the $\mathrm{Ag} / \mathrm{AgX}$ solid solution reference should be merged in seawater for one or two days. It has two aims: One is to estimate the reference electrode with three saturated calomel electrodes (SCE) for ensuring its stability. The other is to activate the $\mathrm{Ag} / \mathrm{AgX}$ solid solution reference electrode, for only activated $\mathrm{Ag} / \mathrm{AgX}$ solid solution reference electrodes have good performances when measuring potential and can be used in actual measurement.

\subsection{Development of the acquisition card}

For the purpose of getting accurate data of corrosion potential in electrolyte, adequate consideration of the 
characteristics of electric field as well as the polarization property of $\mathrm{Ag} / \mathrm{AgX}$ solid solution reference electrode are given before design of the measurement hardware system. The following requirements should be satisfied to ensure the reliability of the data acquisition hardware system:

- The input impedance of data acquisition equipment should be greater than $10 \mathrm{G}$ ohm.

- The minimum detectable voltage of data acquisition equipment must be less than $0.1 \mathrm{mv}$.

- The maximum error should be less than $0.1 \mathrm{mv}$ in the whole measurement range.

- The measurement range should be greater or at least equal to $-1.2 \mathrm{~V} \sim+1.2 \mathrm{~V}$

- The hardware system should have strong antiinterference capacity to adapt to the complex marine environments.

In order to satisfy with the demands above, low-pass filter circuit and signal amplification circuit is employed to act as the signal modulate circuit to improve the input impedance and filter high frequency noise, thereby restraining the noise and enhancing the signal noise ratio effectively. ADS1256 is used as the AD transformation module to ensure the accuracy of data acquisition circuit, for ADS1256 is an extremely low-noise, analog-to-digital (A/D) converters with 24-bit precision. Moreover, a flexible input multiplexer can handle differential or single-ended signals, and a special circuitry can verify the integrity of the external sensor connected to the inputs [13]. In addition, STM32107 is served as the control chip for its high performance price ratio, much greater execution speed, lower power consumption, and larger and more flexible memory model [14]. The frame of the designed hardware system is shown in Figure 1.

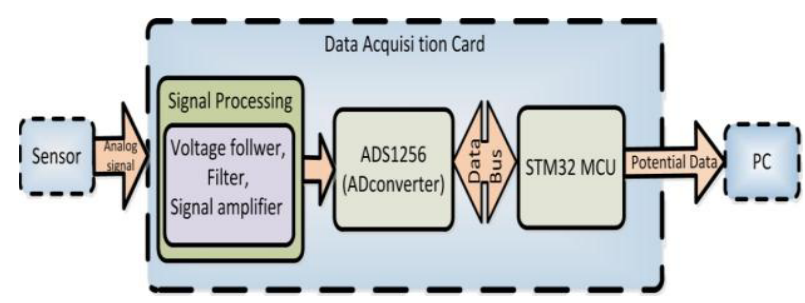

Figure 1. The whole frame of hardware system.

During the practical measurement process, the analogue signals of corrosion potential measured by the potential potentiometric sensor are transmitted to the signal modulate circuit in acquisition card through shielded cable in form of differential input. Then the AD transformation module converts the analogue signal to digital signal, and finally the control chip transmits the obtained potential data to the upper PC through RS-485 bus.

\subsection{Development of the experimental platform}

In order to verify the accuracy of the measuring system, we designed a corrosion simulation experiment platform of submarine pipeline as showed in Figure 2.

The simulating pipeline is an iron rod, with length being $4.8 \mathrm{~m}$, diameter being $10 \mathrm{~cm}$ and is connected with a plate-shaped anode in order to simulate CP system. In addition, the pipeline is coated with a layer of insulating material on the surface and hanged in the seawater by a string. For simulating the corrosion state, a coating defect with length being $15 \mathrm{~cm}$ is made deliberately. The sink is filled with seawater from shore in Qingdao, which conductivity has diluted from $4.252 \mathrm{~S} / \mathrm{m}$ to $1.037 \mathrm{~S} / \mathrm{m}$. According to the relationship in Table 1 we can get the linear scaling factor of this experimental platform: $p=\sigma / \sigma^{\prime}=4.1$. Ulteriorly, that means the spatial scale of prototype is 4.1 times of model according to DACS physical scale model theory.

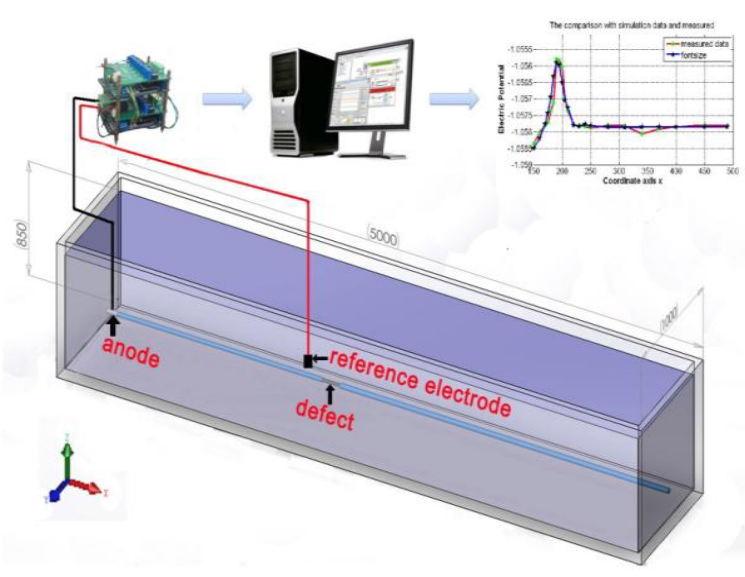

Figure 2. Schematic diagram of the simulated platform.

Then the potential distribution around the simulating pipeline can be measured by the dedicated measuring device developed in this paper as showed in Figure 2. Typically, we measured 3 groups of potential data along the axis direction of simulating pipeline with the vertical distance to pipeline being $0 \mathrm{~cm}, 10 \mathrm{~cm}$ and $25 \mathrm{~cm}$ separately. In addition to manual measuring the potential data in the laboratory sink, we also preformed numerical simulation with FEM model to get the theoretical value of potential of the same points as we measured by hand. Then we can judge whether this set of measuring system is reliable or not by contrasting this two kinds of data.

\section{Experiment result}

The comparison result of the measurement data with the simulation data is shown in Figure 3, where the red solid line represents the simulation data and the blue dotted line represents the measurement data, $\mathrm{x}$ axis represents the extended direction of pipeline beginning with anode, $y$ axis represents the distance between the potential sensor and the pipeline, and $\mathrm{z}$ axis represents the potential values of measured points.

The statistic results of the above comparison result in Figure 4 show that the maximal difference value between the measurement potentials and the calculated potentials of FEM is less than $0.5 \mathrm{mv}$, where 92 percent of the differences are less than $0.25 \mathrm{mv}$, and 86 percent of the differences are less than $0.2 \mathrm{mv}$. That means the measuring system developed in this paper can accurately measure the potentials in the electrolyte environment and 
can be applied to actual marine environment for monitoring subsea pipelines under $\mathrm{CP}$.

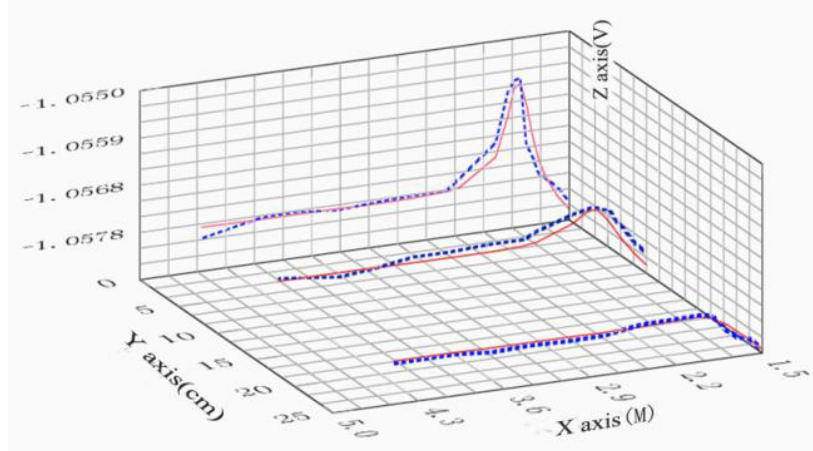

Figure 3. Comparison results between the measured data and FEM

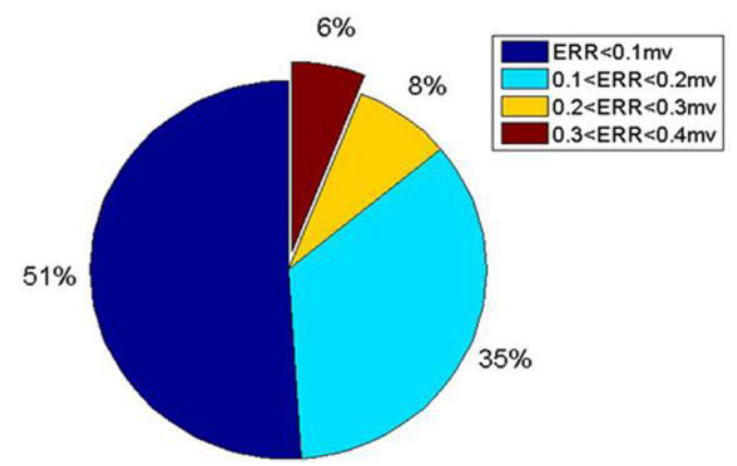

Figure 4. Percentage of measurement error for different level

\section{Conclusions}

In this work we generalized DACS physical scale model from ICCP system to CP system therefore providing a theoretical basis for the simulation of corrosion electric field of large space in a small space and developed a dedicated measuring system then verified the feasibility of the measuring system through simulation experiment. The results demonstrate that the developed measuring system can accurately measure the potentials in the electrolyte environment and can be applied to actual marine environment for monitoring subsea pipelines under CP providing an indirect but more economical and efficient method of measuring potential around submarine pipelines. In the future we will develop a multiple channels electric potential measuring system, which can measure several points concurrently; on that base we can study the space corrosive electric field in profile not line any longer.

\section{References}

1. $\mathrm{Xu}$ LK, Ma L, Xing $\mathrm{SH}$, Review on cathodic protection for marine structure, MATERIALS CHINA 33(2): 106-113 (2014)
2. Jezmar J, Monitoring methods of cathodic protection of pipelines, J.Corros. Meas 2(2): 1-13(2002)

3. Wyatt BS, Cathodic protection monitoring and survey requirements for offshore platforms and pipelines: Part 3, Anti-Corrosion Methods and Materials 32(8): 7-11(1985)

4. Marcassoli P, Bonetti A, Lazzari L, Ormellese M, Modeling of potential distribution of subsea pipeline under cathodic protection by finite element method, Materials and Corrosion(2014)

5. Parks A.R, DAIL THOMAS E, Lucas K.E, Physical scale modeling verification with shipboard trials, Materials performance 30(5): 26-29 (1991)

6. Liang $\mathrm{CH}, \mathrm{Yu} \mathrm{N}, \mathrm{Wu} \mathrm{JH}$, Huang NB, Research on physical scale model of ICCP systems in double zones of hull, Journal of Dalian University of Technology 5(48): 656-660 (2008)

7. DeGiorgi V.G, Thomas III E.D, Lucas K.E, Scale effects and verification of modeling of ship cathodic protection systems, Engineering analysis with boundary elements 22(1): 41-49(1998)

8. Wang Y, KarisAllen KJ, Comparison of Impressed Current Cathodic Protection Numerical Modeling Results with Physical Scale Modeling Data, Corrosion 66(10):105001-105001 (2010)

9. Ditchfield R.W, McGrath J.N, Tighe-Ford D.J, Theoretical validation of the physical scale modelling of the electrical potential characteristics of marine impressed current cathodic protection, Journal of applied electrochemistry 25(1):54-60 (1995)

10. Gao RJ, Du M, Marine corrosion and protection technology, 1rd edn, Chemical Industry Press (CIP), China, pp142-144 (2011)

11. Wang QZ, Siliver-Hlgen Silver Solid Solution Reference Electrode in Seawater, Oceanologia Et Limnologia Sinica 22(4):347-351 (1991)

12. Hu G, Zhang ST, Xiang B, Non-contact detection of the rotted points on the coating surface of the undersea pipelines, Journal of Chongqing University 31(12): 1415-1419 (2008)

13. Zhou C, Li CM, Principle and application of new type8-channel24-bit $\Delta-\Sigma$ analog-to-digital (A/D) converter ADS1256, International Electronic Elements 6:35-38 (2005)

14. Cai JL, Li G, Bu XL, Liu XG, Xing HL, The design of landslide hazard remote monitoring system based on STM32 MCU and GPRS, In Applied Mechanics and Materials 599:1115-1119(2014) 